\title{
Causal effect of alcohol use on the risk of end-stage kidney disease and related comorbidities: a Mendelian randomization study
}

\author{
Sehoon Park ${ }^{1,2}$, Soojin Lee ${ }^{3}$, Yaerim Kim ${ }^{4}$, Yeonhee Lee $^{3}$, Min Woo Kang ${ }^{5}$, Kwangsoo Kim ${ }^{6}$, Yong Chul Kim ${ }^{5}$, \\ Seung Seok Han ${ }^{5,8}$, Hajeong Lee ${ }^{5,8}$, Jung Pyo Lee, ${ }^{7,8,9}$, Kwon Wook Joo ${ }^{5,7,8}$, Chun Soo Lim ${ }^{7,8,9}$, Yon Su Kim ${ }^{1,5,7,8}$, \\ Dong Ki Kim ${ }^{5,7,8}$ \\ ${ }^{1}$ Department of Biomedical Sciences, Seoul National University College of Medicine, Seoul, Republic of Korea \\ ${ }^{2}$ Department of Internal Medicine, Korean Armed Forces Capital Hospital, Seongnam, Republic of Korea \\ ${ }^{3}$ Division of Nephrology, Department of Internal Medicine, Uijeongbu Eulji University Medical Center, Seongnam, Republic of Korea \\ ${ }^{4}$ Department of Internal Medicine, Keimyung University School of Medicine, Daegu, Republic of Korea \\ ${ }^{5}$ Department of Internal Medicine, Seoul National University Hospital, Seoul, Republic of Korea \\ ${ }^{6}$ Transdisciplinary Department of Medicine and Advanced Technology, Seoul National University Hospital, Seoul, Republic of Korea \\ ${ }^{7}$ Department of Internal Medicine, Seoul National University College of Medicine, Seoul, Republic of Korea \\ ${ }^{8}$ Kidney Research Institute, Seoul National University, Seoul, Republic of Korea \\ ${ }^{9}$ Department of Internal Medicine, SMG-SNU Boramae Medical Center, Seoul, Republic of Korea
}

Background: An inverse observational association between alcohol use and the risk of chronic kidney disease (CKD) or end-stage kidney disease (ESKD) has been reported. The causal effect of alcohol use on the risk of ESKD warrants additional investigation.

Methods: The study was an observational cohort study investigating the UK Biobank and performed Mendelian randomization (MR) analysis. Amounts of alcohol use were collected using a touchscreen questionnaire. In the observational analysis, 212,133 participants without prevalent ESKD were studied, and the association between alcohol use and the risk of prevalent CKD or incident ESKD was investigated. The genetic analysis included 337,138 participants of white British ancestry. For one-sample MR, an analysis based on a polygenic risk score (PRS) was conducted with genetically predicted alcohol intake. The MR analysis investigated ESKD outcome and related comorbidities.

Results: Lower alcohol use was observationally associated with a higher risk of prevalent CKD or incident ESKD. However, the genetic risk of CKD was significantly associated with lower alcohol use, suggesting reverse causation. A higher PRS for alcohol use was significantly associated with a higher risk of ESKD (per units of one phenotypical alcohol drink; adjusted odds ratio of 1.16 [95\% confidence interval, 1.02-1.31]) and related comorbidities, including hypertension, diabetes mellitus, obesity, and central obesity.

Conclusion: The inverse observational association between alcohol use and the risk of CKD or ESKD may have been affected by reverse causation. Our study supports a causal effect of alcohol use on a higher risk of ESKD and related predisposing comorbidities.

Keywords: Alcohol, Chronic kidney disease, End-stage kidney disease, Life style, Mendelian randomization

Received: October 12, 2020; Revised: December 9, 2020; Accepted: December 16, 2020

Editor: Eun Hui Bae, Chonnam National University, Gwangju, Republic of Korea

Correspondence: Dong Ki Kim

Department of Internal Medicine, Seoul National University College of Medicine, 101 Daehak-ro, Jongno-gu, Seoul 03080, Republic of Korea. E-mail:dkkim73@gmail.com

ORCID: https://orcid.org/0000-0002-5195-7852

Copyright (C) 2021 by The Korean Society of Nephrology

(a) This is an Open Access article distributed under the terms of the Creative Commons Attribution Non-Commercial and No Derivatives License (http:// creativecommons.org/licenses/by-nc-nd/4.0/) which permits unrestricted non-commercial use, distribution of the material without any modifications, and reproduction in any medium, provided the original works properly cited. 


\section{Introduction}

End-stage kidney disease (ESKD), the terminal state of chronic kidney disease (CKD), is a major and global health problem $[1,2]$. As the prevalence of ESKD is increasing rapidly as populations' age, the socioeconomic burden of ESKD is growing, with patients suffering from a heightened risk of death and impaired quality of life.

Alcohol use is a leading modifiable lifestyle factor associated with higher risks of mortality and various disabilities. Alcohol use is also prevalent in individuals with CKD, and heavy alcohol consumption has been identified in a non-negligible portion of individuals with impaired kidney function [3]. However, debate persists regarding the possible protective associations of moderate alcohol consumption with respect to some clinical conditions [4]. Similarly, moderate alcohol use was associated with a lower risk of CKD or ESKD in several previous reports [5-8] and in a meta-analysis of prospective cohorts [9]. In addition, light-to-moderate alcohol use is not discouraged by current guidelines for CKD patients [10]. However, as this inverse association between alcohol use and CKD or ESKD may mislead the public into assuming that alcohol use is relatively safe for the kidney, additional explanation of the clinical association is necessary. Such observational findings can be affected by reverse causation, particularly when studying a lifestyle factor that is recognized to be harmful to health. Thus, additional investigations of the causal effects of alcohol use on the risk of ESKD or related comorbidities are warranted.

Mendelian randomization (MR) is a useful tool to assess the causal effects of a modifiable environmental factor on complex diseases in observational cohorts [1]. Because the genetic instrument implemented in MR is determined inborn, the genetic exposure is minimally biased by reverse causation or confounding. The method has been used to suggest a causal linkage between alcohol use and the risk of cardiovascular disease as part of efforts to correct misunderstanding of the purported benefits of light-to-moderate alcohol use on cardiovascular health [11].

In this study, we assessed the causal effects of alcohol use on the risk of ESKD or related comorbidities in a UK Biobank prospective cohort. An inverse observational association between alcohol use and prevalent CKD was present in the studied cohort, but an MR analysis revealed that this may be the result of reverse causation. We also used MR to demon- strate that higher alcohol use may increase the risk of ESKD or related comorbidities, including hypertension, diabetes mellitus, or obesity.

\section{Methods}

\section{Ethical considerations}

The study was performed in accordance with the Declaration of Helsinki and approved by the Institutional Review Boards of Seoul National University Hospital (No. E-2005182-1126) and the UK Biobank Consortium (application No. 53799). The requirement for informed consent was waived because we used data from an anonymized public database.

\section{Study setting}

The UK Biobank is a population-scale prospective cohort that recruited $>500,000$ participants aged 40 to 69 years from multiple centers in the UK from 2006 to 2010 . The project assessed various socioeconomic and lifestyle factors and laboratory and anthropometric measurements. Details of the UK Biobank database have been previously described [12].

\section{Study population}

For the observational analysis, we screened 502,505 UK Biobank participants at the time of study approval (Fig. 1). We excluded those for whom information on phenotypic alcohol amounts was missing ( $n=162,223)$, those without information on the studied covariates ( $\mathrm{n}=127,959)$, and those with a previous ESKD history, based on baseline estimated glomerular filtration rate (eGFR) of $<15 \mathrm{~mL} / \mathrm{min} / 1.73 \mathrm{~m}^{2}(\mathrm{n}=190)$, as we were examining incident ESKD cases as an outcome. After exclusion, 212,133 participants with complete information for the studied variables remained.

For the genetic analysis, we applied sample filters using information provided by the UK Biobank and included individuals of European ancestry who were not outliers in terms of heterozygosity or missing data and who had no sex chromosome aneuploidy or excess kinship. After exclusion, 337,138 participants remained for the genetic analysis. 


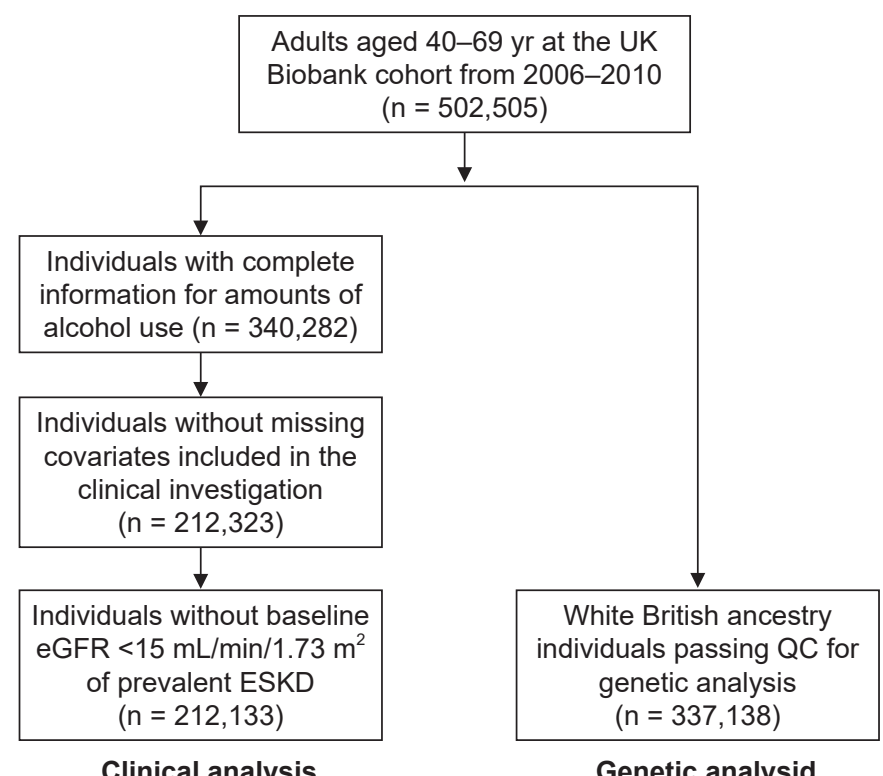

Figure 1. Study population.

eGFR, estimated glomerular filtration rate; ESKD, end-stage kidney disease; QC, quality control.

\section{Alcohol use}

Self-reported amounts of alcohol use were collected using a standardized touchscreen questionnaire. The participants were asked to report their average weekly consumption of specific types of alcoholic drinks. The touchscreen questionnaire was accompanied by pictures of an example of a single unit of each drink type. We summed the self-reported amounts of each drink type to derive the average total alcohol use in units per week. Those with missing data for any of the alcohol types were excluded.

\section{Clinical outcomes}

In the clinical analysis, we first assessed the association between alcohol use exposure and the prevalence of CKD stage $\geq 3$, determined by baseline eGFR, calculated by the Chronic Kidney Disease Epidemiology Collaboration equation, $<60$ $\mathrm{mL} / \mathrm{min} / 1.73 \mathrm{~m}^{2}[10,13]$. Next, we studied the incident ESKD outcomes during follow-ups. Incident ESKD information was algorithmically defined by the UK Biobank, including hospital electronic health records and death registries. The follow-up was censored on February 29, 2016, as complete hospital inpatient data were available only until that date in all three regions (England, Scotland, and Wales).
In the genetic analysis, we studied the total ESKD outcome, both prevalent and incident, of the genotyped participants as a binary variable, as there was no concern that disease occurrence would influence the genetic exposure, in contrast to conventional observational studies [14]. In MR, a genetic instrument can be used regardless of the timing of the outcome and baseline assessment, as the genotype is determined inborn. Details of the collection of the other covariates are described in the Supplementary Methods (available online).

\section{Statistical methods for observational analyses}

Observational associations between the ordinal alcohol use category and prevalent CKD were investigated by logistic regression analysis. The risk of incident ESKD was analyzed by Cox regression analysis. Details of the statistical methods for observational analyses are presented in the Supplementary Methods.

\section{Genetic analysis}

We performed an allele-score MR as a one-sample MR analysis [15]. Two-sample MR was not implemented was because external genome-wide association study (GWAS) summary statistics for ESKD covering sufficient single nucleotide polymorphisms (SNPs) for the approach were absent. Details of the imputation process and genotyping have been described previously [16]. For a GWAS to determine the genetic instrument, we filtered out variants with allele frequency of $<0.1 \%$, and a p-value of $<5 \times 10^{-8}$ was applied to identify significant SNPs associated with the phenotype and the continuous variable for amounts of total alcohol intake, adjusting for age, sex, and the first 20 principal components by linear regression. After removing SNPs in linkage disequilibrium $\left(\mathrm{R}^{2}<0.1\right)$, the genetic instrument for alcohol amounts was determined. Genes containing or related to SNPs were searched using the Ensembl Variant Effect Predictor [17]. We tested whether the SNPs included in the genetic instrument were directly associated with the CKD or ESKD phenotype by GWAS to determine whether a disproportionate effect of an individual SNP would affect the overall results (a single-SNP analysis). If a single SNP reached a Bonferroni-corrected significance level associated with the outcome phenotypes, a reason for the result (e.g., 
presence of a pleiotropic pathway) would need to be identified. We used the genetic instrument to calculate a polygenic risk score (PRS) with the regression effect sizes, betas of the GWAS results, and the gene dosage matrix, and the PRS was regressed to the phenotypes of interest. All of the above processes were performed in PLINK2.0 (version alpha 2.3) [18] and R (version 3.6.2; R Foundation for Statistical Computing, Vienna, Austria) [18]. The instrumental power was estimated by calculating F statistics in the regression model for a PRS of alcohol-intake amount and phenotypical self-reported alcohol intake.

First, we investigated whether there was a reverse causation between CKD and alcohol use-whether individuals with a genetic risk of CKD drink less alcohol. We constructed a genetic instrument for CKD stage $\geq 3$, as determined by an eGFR of $<60 \mathrm{~mL} / \mathrm{min} / 1.73 \mathrm{~m}^{2}$ or a prevalent history of ESKD. The PRS for CKD was linearly regressed to phenotypic amounts of alcohol use, and a multivariable model adjusted for age, sex, hypertension, and diabetes mellitus, which are major factors related to kidney function, was also constructed. As large-scale GWAS results for ESKD were absent due to the low prevalence of the outcome, an analysis including PRS for ESKD was not performed.

Second, we investigated whether genetically predicted amounts of alcohol use were associated with a risk of ESKD or related comorbidities, lifestyles, and socioeconomic status, including CKD stage $\geq 3$, hypertension, diabetes mellitus, obesity, central obesity, current smoking, average days of moderate physical activity, income grade, or the number of household members. The PRS, scaled to reflect one phenotypical unit of average alcohol consumption, was regressed by logistic or linear regression to the phenotypes, and ageand sex-adjusted effect sizes were plotted.

Third, we tested whether alcohol-use phenotype, numerical value, or stratification by the recommendation ( $\leq 2$ drinks for males, $\leq 1$ drink for females per day) [19] interacted with the causal effects of the alcohol-use PRS and risk of ESKD with interaction-term analysis.

Fourth, to minimize the effect of horizontal pleiotropy and test whether alcohol use had a direct causal effect on the risk of ESKD, we constructed a multivariable model adjusted for age, sex, and phenotypes significantly associated with the PRS for amounts of alcohol use.

Fifth, to further assess the independence and exclusion-restriction assumptions, a "negative-control approach" was utilized [1]. If the genetic effect from the PRS for alcohol use was through the phenotypical alcohol-intake amount, the association between a genetic predisposition for exposure and outcomes would be attenuated in those with low amounts of phenotypical alcohol intake. This is because an effect from a higher alcohol-intake trait would be absent in negative controls, and if the significance remained, this means that another pleiotropic pathway rather than phenotypical alcohol intake, mediated the genetic effects. This approach has been suggested previously to test important MR assumptions when demonstrating causal effects. In this analysis, the main PRS analysis was limited to those in the genetic analysis dataset with an alcohol intake of $\leq 7$ drinks per week.

Finally, as we chose the one-sample MR method, the results may have been biased toward observational findings [20]. Although the direction of clinically observed associations was inverse, we minimized such bias by recalculating the regressed betas for the alcohol-amount phenotype and excluding CKD stage $\geq 3$ cases from the genetic dataset. This approach, which calculates the association between the genetic instrument and the exposure of interest only in the controls, reportedly yields valid estimates of exposure, even in a one-sample MR [20]. We recalculated the PRS, which was again regressed to the ESKD or CKD outcome with multiple adjustments for phenotypes associated with the PRS for amounts of alcohol use.

\section{Results}

\section{Clinical characteristics}

Clinical characteristics according to ordinal categories of amounts of alcohol use are presented in Table 1. There were $3,694,83,392,67,522$, and 57,525 participants who reported consuming an average of 0 or 1 drink, $>1$ and $\leq 7$ drinks, $>7$ and $\leq 14$ drinks, and $>14$ drinks per week, respectively. Those who reported higher amounts of alcohol use were more likely to be male, obese, or centrally obese. Baseline dyslipidemia, hypertension, or current smoking was particularly common in those reporting consuming $>14$ drinks per week. Those with an annual income of $<£ 18,000$ were more common among those who reported 0 or 1 drink per week, but those who reported earning $£ 52,000$ to $£ 100,000$ or $>£ 100,000$ per year were more common among those who consumed larg- 
Table 1. Baseline characteristics according to self-reported amounts of alcohol intake per week

\begin{tabular}{|c|c|c|c|c|}
\hline \multirow{2}{*}{ Characteristic } & \multicolumn{4}{|c|}{ Alcohol intake (drink/wk) } \\
\hline & 0 or 1 & $>1$ and $\leq 7$ & $>7$ and $\leq 14$ & $>14$ \\
\hline No. of participants & 3,694 & 83,392 & 67,522 & 57,525 \\
\hline Alcohol use (time/wk) & $1(1-1)$ & $5(3-6)$ & $10(9-12)$ & $21(17-28)$ \\
\hline Age (yr) & $59(51-64)$ & $58(50-63)$ & $58(50-63)$ & $58(51-63)$ \\
\hline \multicolumn{5}{|l|}{ Sex } \\
\hline Female & $2,406(65.1)$ & $49,677(59.6)$ & $30,560(45.3)$ & $16,129(28.0)$ \\
\hline Male & $1,288(34.9)$ & $33,715(40.4)$ & $36,962(54.7)$ & $41,396(72.0)$ \\
\hline Body mass index $\left(\mathrm{kg} / \mathrm{m}^{2}\right)$ & $25.9(23.4-29.2)$ & $26.0(23.6-29.0)$ & $26.4(24.0-29.2)$ & $27.2(24.8-29.9)$ \\
\hline Obesity $^{a}$ & $760(20.6)$ & $15,827(19.0)$ & $13,400(19.8)$ & $14,157(24.6)$ \\
\hline Waist circumference (cm) & $86(77-96)$ & $87(78-96)$ & $90(81-98)$ & $94(86-102)$ \\
\hline Central obesity $^{\mathrm{b}}$ & $1,119(30.3)$ & $23,373(28.0)$ & $19,173(28.4)$ & $19,377(33.7)$ \\
\hline $\begin{array}{l}\text { Previous history of stroke, angina, } \\
\text { or heart attack }\end{array}$ & $178(4.8)$ & $3,461(4.2)$ & $3,255(4.8)$ & $3,272(5.7)$ \\
\hline Hypertension & $641(17.4)$ & $13,982(16.8)$ & $12,298(18.2)$ & $13,335(23.2)$ \\
\hline Systolic BP (mmHg) & $134.0(122.5-147.5)$ & $134.0(122.5-147.0)$ & $136.0(125.0-148.5)$ & 140.5 (129.0-153.0) \\
\hline Diastolic BP (mmHg) & 80.5 (73.5-87.0) & $81.0(74.5-87.5)$ & $82.0(75.5-89.0)$ & 84.5 (78.0-91.5) \\
\hline Diabetes mellitus & $154(4.2)$ & $3,025(3.6)$ & $2,346(3.5)$ & $2,512(4.4)$ \\
\hline Hemoglobin A1c (mmol/mol) & $35.3(32.9-37.9)$ & $35.0(32.6-37.4)$ & $34.7(32.3-37.2)$ & $34.8(32.3-37.3)$ \\
\hline Dyslipidemia & $566(15.3)$ & $11,534(13.8)$ & $10,613(15.7)$ & $13,335(23.2)$ \\
\hline Total cholesterol (mmol/L) & $5.6(4.9-6.4)$ & $5.7(4.9-6.4)$ & $5.7(5.0-6.4)$ & $5.7(5.0-6.5)$ \\
\hline LDL cholesterol (mmol/L) & $3.5(3.0-4.1)$ & $3.5(3.0-4.1)$ & $3.5(3.0-4.1)$ & $3.5(3.0-4.1)$ \\
\hline HDL cholesterol (mmol/L) & $1.4(1.2-1.7)$ & $1.4(1.2-1.7)$ & $1.4(1.2-1.7)$ & $1.5(1.2-1.7)$ \\
\hline \multicolumn{5}{|l|}{ History of smoking } \\
\hline None & $2,506(67.8)$ & $52,079(62.5)$ & $34,176(50.6)$ & $20,845(36.2)$ \\
\hline Ex-smoker & $990(26.8)$ & $25,977(31.2)$ & $27,155(40.2)$ & $27,159(47.2)$ \\
\hline Current smoker & $198(5.4)$ & $5,336(6.4)$ & 6,191 (9.2) & $9,521(16.6)$ \\
\hline Moderate physical activity (day/wk) & $3(2-5)$ & $3(2-5)$ & $3(2-5)$ & $3(2-5)$ \\
\hline No. of illnesses & $1(0-3)$ & $1(0-2)$ & $1(0-2)$ & $1(0-3)$ \\
\hline No. of treatments received & $2(0-3)$ & $1(0-3)$ & $1(0-3)$ & $2(0-3)$ \\
\hline \multicolumn{5}{|l|}{ Income grade (GBP) } \\
\hline$<18,000$ & $941(25.5)$ & $15,218(18.2)$ & $10,555(15.6)$ & $9,232(16.0)$ \\
\hline $18,000-30,999$ & $1,010(27.3)$ & $21,262(25.5)$ & $16,129(23.9)$ & $13,238(23.0)$ \\
\hline $31,000-51,999$ & $962(26.0)$ & $23,223(27.8)$ & $18,904(28.0)$ & $15,809(27.5)$ \\
\hline $52,000-100,000$ & $659(17.8)$ & $18,980(22.8)$ & $16,818(24.9)$ & $14,487(25.2)$ \\
\hline$>100,000$ & $122(3.3)$ & 4,709 (5.6) & $5,116(7.6)$ & 4,759 (8.3) \\
\hline No. of household member & $2(2-3)$ & $2(2-3)$ & $2(2-3)$ & $2(2-3)$ \\
\hline eGFR (mL/min/1.73 $\left.\mathrm{m}^{2}\right)$ & $91.9(81.8-99.1)$ & $92.3(82.6-99.5)$ & $92.6(83.4-99.6)$ & $93.4(84.6-100.2)$ \\
\hline$<60 \mathrm{~mL} / \mathrm{min} / 1.73 \mathrm{~m}^{2}$ & $103(2.8)$ & $1,588(1.9)$ & $1,115(1.7)$ & $875(1.5)$ \\
\hline
\end{tabular}

Data are expressed as number only, median (interquartile range), or number (\%).

BP, blood pressure; eGFR, estimated glomerular filtration rate; GBP, Great Britain pound; HDL, high-density lipoprotein; LDL, low-density lipoprotein.

aody mass index $\geq 30 \mathrm{~kg} / \mathrm{m}^{2}$. ${ }^{\mathrm{b}} \geq 102 \mathrm{~cm}$ for males, $\geq 88 \mathrm{~cm}$ for females.

er amounts of alcohol. The prevalence of CKD stage $\geq 3$ was $3 \%$ in those with 0 or 1 drink per week of alcohol use, which was higher than the other groups.

\section{Obervational association between alcohol use and CKD or ESKD}

Those who reporting consuming 0 or 1 alcoholic drink per week had more than $30 \%$ greater odds of being diagnosed 
with prevalent CKD than those who reported consuming $>1$ and $\leq 7$ drinks per week (Table 2). Even among those who reported higher amounts of alcohol use, the odds for prevalent CKD were significantly lower, and this remained similar in multivariable models adjusted for various clinical, lifestyle, and socioeconomic factors. During a median of 7.0 years (interquartile range, 6.3-7.6 years) of follow-up, 135 participants progressed to ESKD in the clinical analysis dataset. Individuals consuming 0 or 1 drink per week had an approximately 3-fold risk of ESKD compared with those who reported $>1$ and $\leq 7$ drinks per week of alcohol use, even after adjustment for multiple variables.

\section{Reverse causation of genetically predicted CKD on amounts of alcohol use}

The analysis revealed 849 SNPs with GWAS-significant p-val- ues for the CKD stage $\geq 3$ phenotype. After removing those with an $\mathrm{R}^{2}$ value of $<0.1$, a total of 17 SNPs were identified as the genetic instrument for CKD (Supplementary Table 1 , available online). When we regressed the PRS for CKD to the amounts of alcohol use, a higher PRS was significantly associated with lower phenotypical amounts of alcohol use, implying that the presence of CKD is a causative factor for low phenotypical alcohol use amount (Table 3).

\section{Genetically predicted amounts of alcohol use and risk of ESKD and related factors}

A total of 4,596 SNPs with GWAS-significant p-values were observed for the numerical amounts of alcohol-use phenotype. The 35 SNPs not in a linkage disequilibrium state $\left(\mathrm{R}^{2}<\right.$ 0.1 ) were identified as the genetic instrument for genetically predicted amounts of alcohol use (Table 4). The genetic in-

Table 2. Risk of prevalent CKD or incident ESKD according to amounts of alcohol use

\begin{tabular}{|c|c|c|c|c|c|c|}
\hline \multirow{2}{*}{ Alcohol use (drink/wk) } & \multicolumn{2}{|c|}{ Univariable model } & \multicolumn{2}{|l|}{ Multivariable model 1} & \multicolumn{2}{|l|}{ Multivariable model 2} \\
\hline & OR or HR $(95 \% \mathrm{Cl})$ & p-value & Adjusted OR or HR (95\% Cl) & p-value & Adjusted OR or HR (95\% Cl) & $p$-value \\
\hline \multicolumn{7}{|l|}{ Prevalent CKD } \\
\hline 0 or 1 & $1.48(1.21-1.81)$ & $<0.001$ & $1.38(1.13-1.70)$ & $<0.001$ & $1.31(1.06-1.61)$ & 0.01 \\
\hline$>1$ and $\leq 7$ & Reference & & Reference & & Reference & \\
\hline$>7$ and $\leq 14$ & $0.87(0.80-0.93)$ & $<0.001$ & $0.86(0.79-0.93)$ & 0.002 & $0.89(0.82-0.97)$ & 0.005 \\
\hline$>14$ & $0.80(0.73-0.87)$ & $<0.001$ & $0.73(0.67-0.79)$ & $<0.001$ & $0.80(0.73-0.87)$ & $<0.001$ \\
\hline \multicolumn{7}{|l|}{ Incident ESKD } \\
\hline 0 or 1 & 2.95 (1.26-6.89) & 0.010 & $2.62(1.12-6.14)$ & 0.03 & $3.17(1.34-7.50)$ & 0.009 \\
\hline$>1$ and $\leq 7$ & Reference & & Reference & & Reference & \\
\hline$>7$ and $\leq 14$ & $1.15(0.76-1.73)$ & 0.52 & $1.38(0.91-2.09)$ & 0.13 & $1.40(0.92-2.15)$ & 0.12 \\
\hline$>14$ & $1.17(0.76-1.79)$ & 0.48 & $1.29(0.83-1.99)$ & 0.25 & $1.18(0.75-1.86)$ & 0.47 \\
\hline
\end{tabular}

For the prevalent CKD outcome, logistic regression analysis was performed (OR), and for the incident ESKD outcome, Cox regression analysis was performed (HR).

Multivariable model 1 was adjusted for age, sex, history of diabetes mellitus, and hypertension. When analyzing the incident ESKD outcome, the baseline eGFR was additionally adjusted.

Multivariable model 2 was adjusted for age, sex, body mass index, waist circumference, history of angina/heart attack/stroke, diabetes mellitus, hemoglobin A1c level, hypertension, systolic blood pressure (BP), diastolic BP, dyslipidemia, total cholesterol, low-density lipoprotein cholesterol, highdensity lipoprotein cholesterol, smoking (nonsmoker, ex-smoker, current smoker), average days of moderate physical activity per week, number of illnesses,

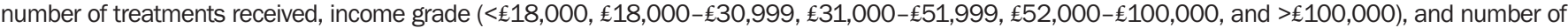
household members.

$\mathrm{Cl}$, confidence interval; CKD, chronic kidney disease; ESKD, end-stage kidney disease; HR, hazard ratio; OR, odds ratio.

Table 3. Genetic predisposition to chronic kidney disease and its association with alcohol-intake phenotype

\begin{tabular}{|c|c|c|c|c|}
\hline & \multicolumn{2}{|c|}{ Univariable model } & \multicolumn{2}{|c|}{ Multivariable model } \\
\hline & $\operatorname{Exp}(\beta)(95 \% \mathrm{Cl})$ & p-value & Adjusted $\exp (\beta)(95 \% \mathrm{Cl})$ & p-value \\
\hline For numerical amounts of alcohol intake & $0.96(0.92-0.99)$ & 0.04 & $0.95(0.92-0.99)$ & 0.02 \\
\hline
\end{tabular}

Reported $\exp (\beta)$ and confidence interval values were from a linear regression model with amounts of alcohol use as the outcome variable and polygenic risk score (PRS) for chronic kidney disease stage $\geq 3$ as the exposure variable. The effect sizes of one standard deviation increment of the PRS are reported. The multivariable model was adjusted for age, sex, diabetes mellitus, and hypertension.

$\mathrm{Cl}$, confidence interval. 
strument included SNPs annotated to genes, such as those for alcohol dehydrogenase $1 \mathrm{~B}(A D H 1 B)$ or 1C $(A D H 1 C)$, which are related to alcohol-use behavior. The SNPs included in the genetic instrument $(0.05 / 35)$ did not reach a Bonferroni-adjusted significant $p$-value when the associations between individual SNPs and CKD stage $\geq 3$ or ESKD pheno- type were investigated (Supplementary Table 2, 3; available online).

The PRS for alcohol intake was significant $\left(\mathrm{p}<2 \times 10^{-16}\right)$ for the self-reported alcohol intake phenotype, and the F statistic was 972.8. The PRS therefore had sufficient instrumental power to test the causal estimates from alcohol intake on

Table 4. Genetic instrument for the calculation of polygenic risk score of amounts of alcohol intake

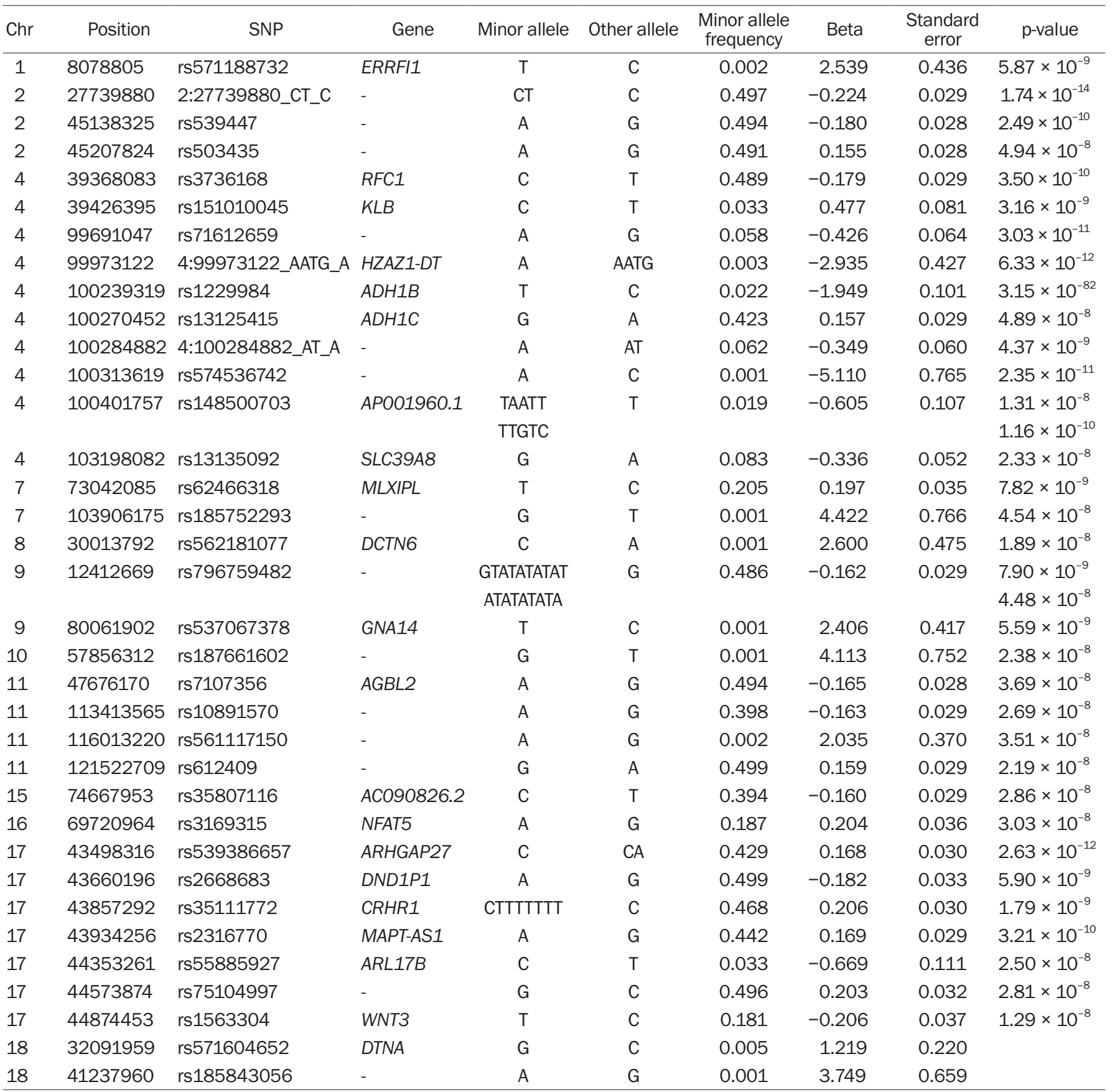

Chr, chromosome; SNP, single nucleotide polymorphism. 


\section{kidney function traits.}

Higher genetically predicted amounts of alcohol use were significantly associated with a higher risk of ESKD, hypertension, diabetes mellitus, obesity, central obesity, current smoking, and a lower number of household members (Fig. 2). The association between the PRS and CKD stage $\geq 3$ phenotype did not reach statistical significance, although the odds ratio $[\mathrm{OR}]$ was above 1 . The average number of days per week of moderate physical and income grades were not significantly associated with the PRS for amounts of alcohol use.

In addition, there was no significant interaction between phenotypical amounts of alcohol use and the association with genetically predicted amounts of alcohol use and the risk of CKD (interaction $\mathrm{p}=0.20$ ) or ESKD (interaction $\mathrm{p}$ $=0.81)$. When alcohol use within the recommended level (an average of $\leq 2$ drinks daily for males or $\leq 1$ drink daily for females) was tested, the variable did not show a significant interaction with the association with CKD (interaction $\mathrm{p}=$ 0.996) or ESKD (interaction $p=0.27$ ).

In the multivariable model adjusted for baseline age, sex, hypertension, diabetes mellitus, obesity, central obesity, current smoking, and lower number of household members, the genetically predicted amounts of alcohol use were still significantly associated with a higher risk of ESKD (per unit reflecting one phenotypical alcohol-amount use: adjusted OR, 1.16; 95\% confidence interval [CI], 1.02-1.31; $\mathrm{p}=0.02$ ), implying a direct effect of alcohol use on the ESKD risk.

In the negative-control approach, the genetic effect from the alcohol intake PRS to ESKD was attenuated in those with low amounts of phenotypical alcohol intake, implying that alcohol-intake exposure mediated the genetic effect to ESKD

\section{Polygenic risk score for amounts of alcohol use}

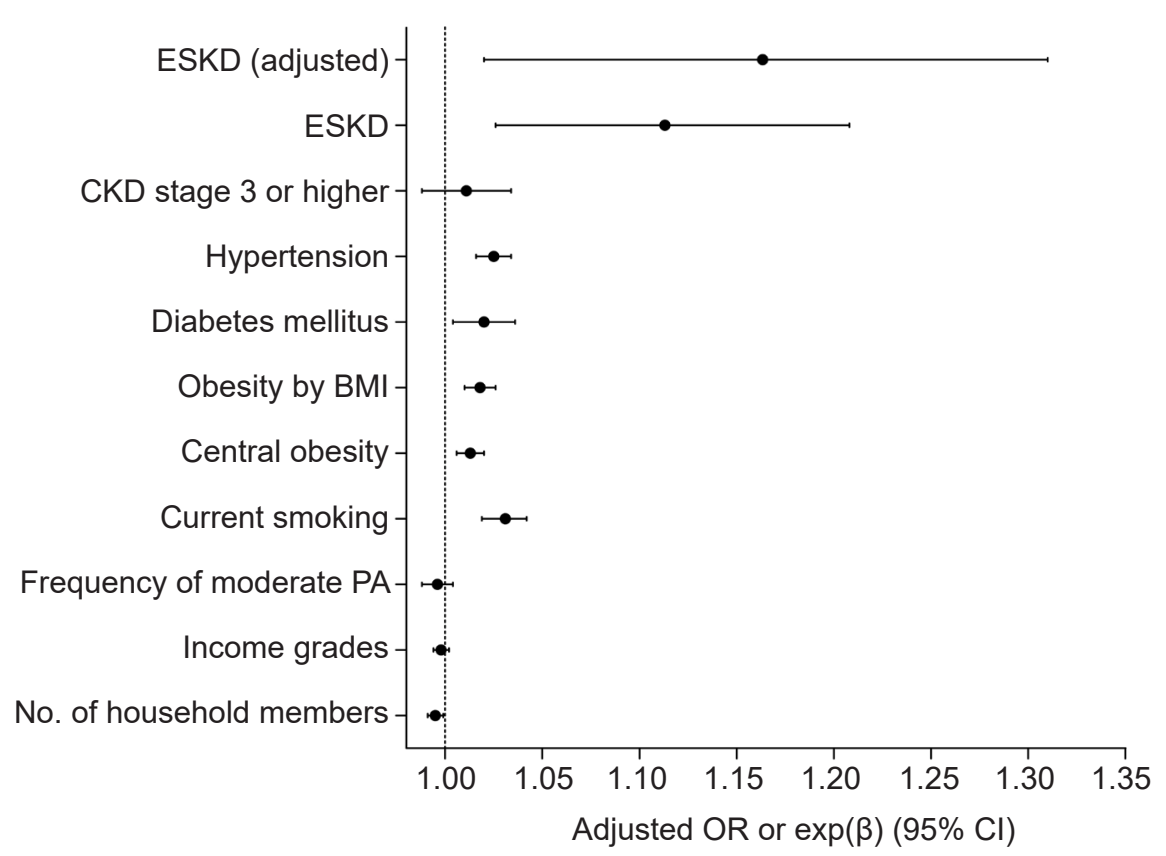

Figure 2. A higher polygenic risk score for amounts of alcohol use was associated with a higher risk of ESKD and related comorbidities.

Multivariable logistic or linear regression analysis was performed with the calculated polygenic risk scores based on 35 single nucleotide polymorphisms, and the age- and sex-adjusted effect sizes per unit of polygenic risk score scaled to reflect one phenotypical unit of alcohol consumption (continuous) increase are plotted. For the ESKD (adjusted) outcome, all identified phenotypes that were significantly associated with the polygenic risk score for alcohol amounts, including age, sex, hypertension, diabetes mellitus, obesity, central obesity, current smoking, and lower number of household members, were adjusted for the multivariable model, implying a direct effect from genetical predisposition for alcohol use amount to risk of ESKD. The dots indicate the odds ratio (OR) or exp( () , and the horizontal lines indicate the $95 \%$ confidence interval $(\mathrm{Cl})$.

BMI, body mass index; CKD, chronic kidney disease; ESKD, end-stage kidney disease; PA, physical activity. 
(Supplementary Table 4, available online). The PRS for alcohol intake remained insignificantly associated with the risk of CKD, but as the main analysis results were not significant, this result could not be interpreted.

Finally, when we obtained regressed betas for amounts of alcohol use of the genetic instrument only in those without CKD stage $\geq 3$, the recalculated PRS was still significantly associated with a higher risk of ESKD (adjusted OR, 1.16; 95\% CI, 1.02-1.31; $\mathrm{p}=0.03$ ), but still not with a risk of CKD (adjusted OR, 1.00; 95\% CI, 0.97-1.04; $\mathrm{p}=0.99$ ).

\section{Discussion}

The inverse association revealed in this study between alcohol use and CKD could have been affected by reverse causation. Furthermore, genetically predicted higher amounts of alcohol use were significantly associated with the risk of ESKD and related factors, including hypertension, diabetes mellitus, obesity, central obesity, current smoking, and a low number of household members. The MR results support recommendations that alcohol be avoided, as it causally elevates the risk of ESKD and related comorbidities.

Our study showed that higher amounts of alcohol use can cause important predisposing comorbidities for CKD, including obesity, hypertension, or diabetes mellitus. The risk of ESKD was significantly higher according to higher genetically predicted amounts of alcohol use, and this association was not affected by whether alcohol use was within the recommended level or the amounts of alcohol-use phenotype. The MR results suggest a causal link between higher amounts of alcohol use and an increased risk of ESKD and that the use of alcohol may generally increase the risk of ESKD [1]. As recent evidence has shown that there is no "safe" level of alcohol use [2], this may be a reasonable recommendation for people overall or individuals with CKD, not only regarding the risk of ESKD but also considering the risk of general mortality.

Our analysis including the PRS is a one-sample MR method, and MR requires three assumptions to be met to suggest causal effects [21]. First, the genetic instrument should be strongly associated with the studied outcome. As the instrument was obtained from a GWAS, the assumption was met. Second, the genetic instrument should affect the outcome through the exposure of interest. Third, the association should not be the result of horizontal pleiotropy, implying the presence of confounders. We found that important comorbidity and lifestyle factors related to the risk of ESKD were significantly associated with the PRS for alcohol use. As the association between the PRS and risk of ESKD remained significant even after adjusting for comorbidities, the finding may not be the result of horizontal pleiotropy but possibly of vertical pleiotropy, which does not violate the MR assumption. Alcohol use may cause diabetes mellitus, hypertension, obesity or central obesity, or current smoking behavior, and, as one of the downstream endpoints from the comorbidities, ESKD. The effect was significant even after adjusting for the comorbidities, implying a direct causal effect from alcohol use on ESKD. In addition, the negative-control approach supported the absence of a horizontal pleiotropic pathway, suggesting the attainment of the important MR assumptions.

Previous studies have reported that alcohol can damage kidneys through increased oxidative stress injury associated with ethanol exposure [22-24]. In addition, heavy ethanol consumption reportedly leads to pathologic glomerular changes through activation of the renin-angiotensin-aldosterone pathway or blood pressure increments in vivo [25,26]. As the biological benefit of alcohol use is unlikely to present, the inverse observational association between alcohol use and the risk of CKD, which cannot be simply accepted to reflect causal effects, required further examination. Although few studies suggest that alcohol use over the recommended level elevates the risk of CKD or ESKD [27], not only moderate but also high amounts of alcohol use have been associated with a lower risk of incident CKD or ESKD in several reports $[5-7,28]$ involving multiple ethnic backgrounds $[5,6,28]$, including healthy individuals [7] and those with a certain proportion of metabolic risk factors [5]. However, as even a single drink of alcohol is not safe with respect to the risk of mortality [2], an explanation for the inverse association is necessary to prevent misunderstandings of this observational association. For cardiovascular diseases, adverse causal effects of alcohol use on cardiovascular health have been demonstrated [11]. Our study indicates that CKD may reduce alcohol use; that is, people with a risk of CKD use lower amounts of alcohol. This suggests that the observational inverse association between alcohol use and the risk of CKD should be interpreted simply as those at risk for CKD tend to use less alcohol. Also, the previous observational findings were likely affected by various unmeasured clinical confounding effects (e.g., smoking, drug use, diet, medical 
compliance, or healthy behaviors) $[3,28,29]$, and not discouraging alcohol intake because the risk of kidney function based on such observational results would be misleading.

Our study has several limitations and raises some questions. First, a CKD stage $\geq 3$ outcome was not significantly associated with the PRS for alcohol use. This may be related to the fact that genetic instruments can explain only some of the impacts of the studied factor or that the effect size was too small to be detected in our study, which included a general population with a low prevalence of CKD. Furthermore, the assumption that alcohol use is associated with kidney hyperfiltration may have caused the null result [30]. As diabetes mellitus, hypertension, and obesity are important predisposing factors for CKD, and alcohol use is causally associated with these phenotypes, the result may not be misinterpreted. Second, the result has not been replicated by a two-sample MR. However, as no external large-scale GWAS for ESKD is available, and the issue of reverse causation or hyperfiltration remains for CKD outcomes, the current one-sample MR should be considered an appropriate method for this study. Third, as the information on alcohol use was self-reported, measurement bias may be present. However, the direction of the bias would not affect the positive finding that alcohol use affects the risk of ESKD, as diseased people would likely report lower amounts of alcohol use. Fourth, the utility of PRS risk estimation is currently limited by its simplicity. Further methodologic advances may be necessary to improve the comprehensiveness and to reduce the uncertainty involved in utilizing the PRS [31]. In addition, validation of the suggested PRS for alcohol-intake amount would be necessary to confirm the generalizability of the instrumented information. Last, the genetic analysis included only those of white British ancestry, undermining the generalizability of the results to other ethnic populations.

In conclusion, alcohol use causally increases the risk of ESKD and related comorbidities. As the issue of reverse causation is present in the form of an inverse association between alcohol use and the risk of CKD, the potential beneficial effects of alcohol use on this complex disease should not be erroneously assumed simply based on observed clinical associations. Healthcare providers may recommend avoiding alcohol use to reduce the risk of ESKD and predisposing comorbidities.

\section{Conflicts of interest}

All authors have no conflicts of interest to declare.

\section{Funding}

This work was supported by the Industrial Strategic Technology Development Program - Development of bio-core technology (10077474, Development of early diagnosis technology for acute/chronic renal failure) funded by the Ministry of Trade, Industry and Energy (Republic of Korea).

\section{Authors' contributions}

\section{Conceptualization: SP, HL, KK, KWJ, DKK}

Data curation: SL, YK, YL, MWK, YCK, SSH, HL, JPL, KWJ, CSL, YSK, DKK

Formal analysis: SP, KK, SL, YK

Funding acquisition: SL, YK, DKK

Investigation: SP, DKK

Methodology: HL, JPL, KWJ, CSL, YSK, DKK

Writing-original draft: All authors

Writing-review \& editing: All authors

All authors read and approved the final manuscript.

\section{ORCID}

Sehoon Park, https://orcid.org/0000-0002-4221-2453

Soojin Lee, https://orcid.org/0000-0001-5633-3961

Yaerim Kim, https://orcid.org/0000-0003-1596-1528

Yeonhee Lee, https://orcid.org/0000-0002-9216-420X

Min Woo Kang, https://orcid.org/0000-0002-9411-3481

Kwangsoo Kim, https://orcid.org/0000-0002-4586-5062

Yong Chul Kim, https://orcid.org/0000-0003-3215-8681

Seung Seok Han, https://orcid.org/0000-0003-0137-5261

Hajeong Lee, https://orcid.org/0000-0002-1873-1587

Jung Pyo Lee, https://orcid.org/0000-0002-4714-1260

Kwon Wook Joo, https://orcid.org/0000-0001-9941-7858

Chun Soo Lim, https://orcid.org/0000-0001-9123-6542

Yon Su Kim, https://orcid.org/0000-0003-3091-2388

Dong Ki Kim, https://orcid.org/0000-0002-5195-7852

\section{References}

1. Davies NM, Holmes MV, Davey Smith G. Reading Mendelian 
randomisation studies: a guide, glossary, and checklist for clinicians. BMJ 2018;362:k601.

2. GBD 2016 Alcohol Collaborators. Alcohol use and burden for 195 countries and territories, 1990-2016: a systematic analysis for the Global Burden of Disease Study 2016. Lancet 2018;392:1015-1035.

3. Bundy JD, Bazzano LA, Xie D, et al. Self-reported tobacco, alcohol, and illicit drug use and progression of chronic kidney disease. Clin J Am Soc Nephrol 2018;13:993-1001.

4. Ronksley PE, Brien SE, Turner BJ, Mukamal KJ, Ghali WA. Association of alcohol consumption with selected cardiovascular disease outcomes: a systematic review and meta-analysis. BMJ 2011;342:d671.

5. Reynolds K, Gu D, Chen J, et al. Alcohol consumption and the risk of end-stage renal disease among Chinese men. Kidney Int 2008;73:870-876.

6. White SL, Polkinghorne KR, Cass A, Shaw JE, Atkins RC, Chadban SJ. Alcohol consumption and 5-year onset of chronic kidney disease: the AusDiab study. Nephrol Dial Transplant 2009;24:2464-2472.

7. Schaeffner ES, Kurth T, de Jong PE, Glynn RJ, Buring JE, Gaziano JM. Alcohol consumption and the risk of renal dysfunction in apparently healthy men. Arch Intern Med 2005;165:1048-1053.

8. Hsu YH, Pai HC, Chang YM, Liu WH, Hsu CC. Alcohol consumption is inversely associated with stage 3 chronic kidney disease in middle-aged Taiwanese men. BMC Nephrol 2013;14:254.

9. Li D, Xu J, Liu F, Wang X, Yang H, Li X. Alcohol drinking and the risk of chronic kidney damage: a meta-analysis of 15 prospective cohort studies. Alcohol Clin Exp Res 2019;43:1360-1372.

10. Kidney Disease: Improving Global Outcomes (KDIGO) CKD Work Group. KDIGO 2012 clinical practice guideline for the evaluation and management of chronic kidney disease. Kidney Int Suppl 2013;3:1-150.

11. Holmes MV, Dale CE, Zuccolo L, et al. Association between alcohol and cardiovascular disease: Mendelian randomisation analysis based on individual participant data. BMJ 2014;349:g4164.

12. Sudlow C, Gallacher J, Allen N, et al. UK biobank: an open access resource for identifying the causes of a wide range of complex diseases of middle and old age. PLoS Med 2015;12:e1001779.

13. Levey AS, Stevens LA, Schmid CH, et al. A new equation to estimate glomerular filtration rate. Ann Intern Med 2009;150:604-612.

14. Daghlas I, Dashti HS, Lane J, et al. Sleep duration and myocardial infarction. J Am Coll Cardiol 2019;74:1304-1314.

15. Burgess S, Thompson SG. Use of allele scores as instrumental variables for Mendelian randomization. Int J Epidemiol 2013;42:11341144.
16. Bycroft C, Freeman C, Petkova D, et al. The UK Biobank resource with deep phenotyping and genomic data. Nature 2018;562:203209.

17. McLaren W, Gil L, Hunt SE, et al. The ensembl variant effect predictor. Genome Biol 2016;17:122.

18. Chang CC, Chow CC, Tellier LC, Vattikuti S, Purcell SM, Lee JJ. Second-generation PLINK: rising to the challenge of larger and richer datasets. Gigascience 2015;4:7.

19. US Department of Health and Human Services; US Department of Agriculture. 2015-2020 Dietary Guidelines for Americans. 8th ed. Washington, DC: US Department of Health and Human Services; 2015.

20. Burgess S, Davies NM, Thompson SG. Bias due to participant overlap in two-sample Mendelian randomization. Genet Epidemiol 2016;40:597608.

21. Sheehan NA, Didelez V, Burton PR, Tobin MD. Mendelian randomisation and causal inference in observational epidemiology. PLoS Med 2008;5:e177.

22. Elkomy NM, Ibrahim IA, Elshazly SM, El-Fayoumi HM. Ameliorative effects of clonidine on ethanol induced kidney injury in rats: potential role for imidazoline-1 receptor. Eur J Pharmacol 2018;824:148-156.

23. Latchoumycandane C, Nagy LE, McIntyre TM. Myeloperoxidase formation of PAF receptor ligands induces PAF receptor-dependent kidney injury during ethanol consumption. Free Radic Biol Med 2015;86:179-190.

24. Latchoumycandane C, Nagy LE, McIntyre TM. Chronic ethanol ingestion induces oxidative kidney injury through taurine-inhibitable inflammation. Free Radic Biol Med 2014;69:403-416.

25. Leal S, Ricardo Jorge DO, Joana B, Maria SS, Isabel SS. Heavy alcohol consumption effects on blood pressure and on kidney structure persist after long-term withdrawal. Kidney Blood Press Res 2017;42:664-675.

26. Yamada Y, Endou M, Morikawa S, Shima J, Komatshzaki N. Lactic acid bacteria isolated from Japanese fermented fish (funa-sushi) inhibit mesangial proliferative glomerulonephritis by alcohol intake with stress. J Nutr Metab 2018;2018:6491907.

27. Perneger TV, Whelton PK, Puddey IB, Klag MJ. Risk of end-stage renal disease associated with alcohol consumption. Am J Epidemiol 1999;150:1275-1281.

28. Hu EA, Lazo M, Rosenberg SD, et al. Alcohol consumption and incident kidney disease: results from the atherosclerosis risk in communities study. J Ren Nutr 2020;30:22-30.

29. Shankar A, Klein R, Klein BE. The association among smoking, heavy drinking, and chronic kidney disease. Am J Epidemiol 2006;164:263271. 
30. Lin M, Su Q, Huang H, et al. Alcohol consumption and the risk for renal hyperfiltration in the general Chinese population. Eur J Clin Nutr 2017;71:500-505.
31. Choi SW, Mak TS, O'Reilly PF. Tutorial: a guide to performing polygenic risk score analyses. Nat Protoc 2020;15:2759-2772. 